
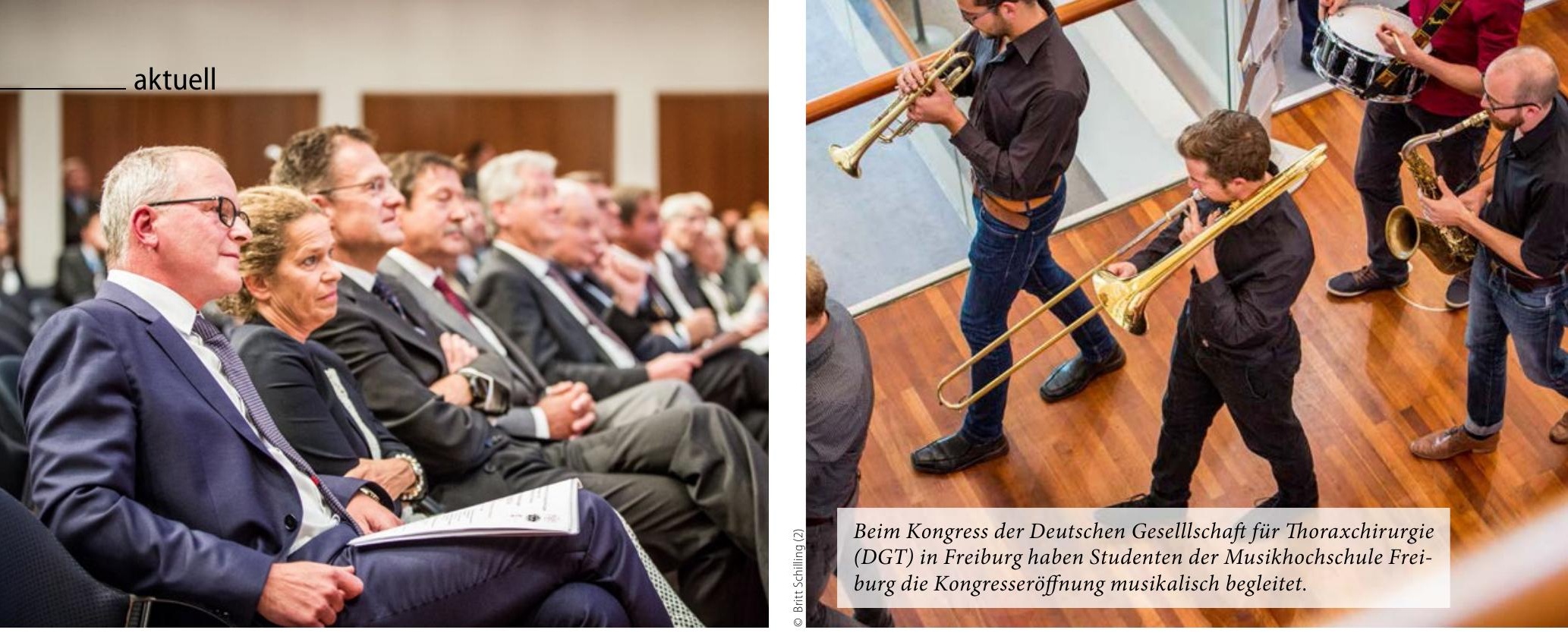

Endobronchiale Navigation

\title{
Das Navi findet den Weg zum Rundherd
}

\author{
Beim Auffinden und Biopsieren sehr kleiner und randständiger Rundherde stößt die konventio- \\ nelle Bronchoskopie oft an ihre Grenzen. Mithilfe der endobronchialen elektromagnetischen Na- \\ vigation kann aber ein Großteil der in der Bildgebung detektierten Rundherde aufgesucht und \\ einer korrekten histologischen Diagnose zugeführt werden. So kann man vielen Patienten die \\ transthorakale Biopsie ersparen, die mit einem hohen Pneumothoraxrisiko einhergeht.
}

„Lungenkrebs ist bei Männern die häufigste, bei Frauen die dritthäufigste Todesursache“, erklärte Dr. Benedikt Haager, Klinik für Thoraxchirurgie, Universitätsklinikum Freiburg. In frühen, lokalisierten Stadien - das heißt zu einem Zeitpunkt, zu dem die Erkrankung am besten behandelbar ist - werden nur $15 \%$ der Fälle diagnostiziert. Die endobronchiale elektromagnetische $\mathrm{Na}$ vigation könnte Haager zufolge zu einer höheren bronchoskopischen Auffindungsrate pulmonaler Herde und somit zu einer höheren Tumorerkennungs- und -resektionsrate beitragen. Das Verfahren basiert auf einer Navigationssoftware und den dreidimensionalen Daten aus einer vor der Bronchoskopie durchgeführten CT. Der Patient liegt während der Bronchoskopie in einem elektromagnetischen Feld, das von einem speziellen Feldgenerator erzeugt wird. Die Bronchoskopspitze enthält neben dem üblichen Biopsieinstrumentarium eine elektromagnetische Lokalisationssonde. Der Computer bestimmt die räumliche Lage der Sonde im Bronchialsystem und berechnet die möglichen Wege zum Rundherd. So kann der Untersucher auch kleine und randständige Rundherde mit der Sonde aufsuchen und biopsieren sowie gegebenenfalls markieren, etwa mit einem Goldseed, um den Herd in einer nachfolgenden Operation leichter wieder aufzufinden.

\section{Hohe diagnostische Aufklärungsrate}

In einer monozentrischen Fallstudie an der Universitätsklinik Salzburg mit 112 Patienten, bei denen zunächst eine singuläre pulmonale Läsion festgestellt worden war, wurde zur weiteren diagnostischen Abklärung die FDG-PET/CT-gestützte endobronchiale elektromagnetische Navigation in Kombination mit Schnellschnitt-Zytologie - „Rapid on-site evaluation“ (ROSE) - eingesetzt. Damit ließ sich bei $76 \%$ aller Läsionen, die im Durchmesser weniger als $20 \mathrm{~mm}$ maßen, eine korrekte pathohistologische Diagnose erreichen. Bei größeren Läsionen lag der diagnostische Yield sogar bei $90 \%$. [1] „Das ist ein erheblicher Fortschritt gegenüber der konventionellen Bronchoskopie mit einem diagnostischen Yield von $53 \%$ ", betonte Haager.

Haager zitiert eine Metaanalyse, die nicht nur einen diagnostischen Yield von über $90 \%$ bestätigt, sondern auch eine mit $2 \%$ sehr niedrige Pneumothoraxrate gezeigt habe, im Vergleich zu etwa $23 \%$ bei CT-gesteuerter transthorakaler Biopsie. [2]

\section{Präoperative Markierung erleichtert das Auffinden}

Ein weiteres viel versprechendes Einsatzgebiet der endobronchialen Navigation sei die Markierung kleiner, schlecht tastbarer Herde vor chirurgischer Resektion. [3] „In Zukunft können wir mithilfe endobronchialer Navigation aufgesuchte Herde möglicherweise gleich behandeln, etwa mit Mikrowellenablation", ergänzte Haager.

Dr. med. Thomas M. Heim

\section{Literatur}

1. Lamprecht B et al. Electromagnetic navigation bronchoscopy (ENB): Increasing diagnostic yield. Respir Med. 2012; 106:710-15.

2. Schreiber G, McCrory DC. Performance characteristics of different modalities for diagnosis of suspected lung cancer: summary of published evidence. Chest 2003; 123(1 Suppl):115-28

3. Andrade RS. Electromagnetic navigation bronchoscopy-guided thoracoscopic wedge resection of small pulmonary nodules. Semin Thorac Cardiovasc Surg 2010;22: 262-65

Quelle: Anästhesiologisch-thoraxchirurgisches Kolloquium am 29.09.2016 im Rahmen der 25. Jahrestagung der Deutschen Gesellschaft für Thoraxchirurgie und 4. D-A-CH-Tagung, Freiburg. 